$\square$ Math-Net.Ru

Общероссийский математический портал 
С. Норвидас, Функциональное исчисление эрмитовых элементов и неравенства Бернштейна, Функи. анализ и его прил., 2006, том 40, выпуск 2, 79-81

DOI: https://doi.org/10.4213/faa10

Использование Общероссийского математического портала Math-Net.Ru 
подразумевает, что вы прочитали и согласны с пользовательским соглашением

http://www.mathnet.ru/rus/agreement

Параметры загрузки:

IP : 54.196.121.252

26 апреля 2023 г., 12:58:14 


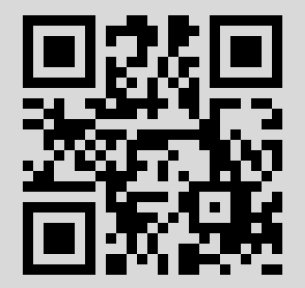


УДК 517.9

\title{
Функциональное исчисление эрмитовых элементов и неравенства Бернштейна
}

\author{
(c) 2006. С. Норвидас
}

Пусть $A$ - банахова алгебра с единицей. Элемент $a \in A$ называется эрмитовым, если $\|\exp (i t a)\|=1$ при $t \in \mathbb{R}$. Норма такого элемента равна его спектральному радиусу, и это утверждение доказывается в рамках стандартного голоморфного функционального исчисления. Для эрмитовых элементов голоморфное исчисление допускает расширение (см. $[1,2])$, которое позволяет описать случаи совпадения нормы и спектрального радиуса для элементов вида $\varphi(a)$ для широкого класса функций $\varphi$ [1]. Мы рассматриваем вопросы такого 
исчисления, связанные с оценками величины $\|\varphi(a)\|_{A}$ через подходящие нормы функции $\varphi$.

Пусть $M(\mathbb{R})$ - банахова алгебра комплексных конечных борелевских мер на $\mathbb{R}$ со сверткой в качестве умножения и полной вариацией в качестве нормы и $B(\mathbb{R})$ - алгебра их преобразований Фурье-Стилтьеса с нормой $\|\widehat{\mu}\|_{B(\mathbb{R})}=$ $\|\mu\|_{M(\mathbb{R})}$. Для эрмитова элемента $a \in A$ и $\varphi=\widehat{\mu} \in B(\mathbb{R})$ можно положить $\varphi(a)=\widehat{\mu}(a)=\int_{\mathbb{R}} \exp ($ iat $) d \mu(t)$. Поскольку спектр $\operatorname{Sp}(a)$ эрмитова элемента $a$ вещественный, из равенства $\varphi_{1}=\varphi_{2}$ в $\mathbb{R}$-окрестности этого спектра вытекает, что $\varphi_{1}(a)=\varphi_{2}(a)$. Поэтому каждый эрмитов элемент $a$ порождает гомоморфизм $\theta_{a}: \varphi \rightarrow \varphi(a)$ алгебры символов $B(\mathbb{R}) / Z(\operatorname{Sp}(a))$ в $A$, где $Z(\operatorname{Sp}(a))$ - замыкание идеала функций из $B(\mathbb{R})$, равных нулю в окрестности спектра $\operatorname{Sp}(a)$. Поскольку $\theta_{a}$ не увеличивает нормы,

$$
\|\varphi\|_{C(\operatorname{Sp}(a))} \leqslant\|\varphi(a)\|_{A} \leqslant\|\varphi\|_{B(\mathbb{R}) / Z(\operatorname{Sp}(a))},
$$

где $C(\operatorname{Sp}(a))$ - обычная алгебра непрерывных на $\operatorname{Sp}(a)$ функций. Равенство $\|\varphi\|_{C(\operatorname{Sp}(a))}=\|\varphi(a)\|_{A}$ означает совпадение нормы и спектрального радиуса элемента $\varphi(a)$. Мы изучим правую оценку в (1) и укажем операторную модель вычисления нормы $\|\varphi\|_{B(\mathbb{R}) / Z(K)}$ для произвольного компакта $K \subset \mathbb{R}$.

Пусть $J(K)$ - идеал тех $\mu \in M(\mathbb{R})$, для которых $\widehat{\mu} \in Z(K)$, а $B_{K}$ - аннулятор идеала $J(K)$ в $L^{\infty}(\mathbb{R})$. Пространство $B_{K}$ состоит из тех функций из $L^{\infty}(\mathbb{R})$, спектр Бьёрлинга которых содержится в $K$. Поэтому элементы из $B_{K}$ можно отождествлять с соответствующими целыми функциями экспоненциального типа. Согласно неравенству Бернштейна, в $B_{K}$ действует эрмитов оператор $D=-i d / d x$. Поскольку $\operatorname{Sp}(D)=K$, каждый символ $\varphi \in B(\mathbb{R}) / Z(K)$ однозначно определяет на $B_{K}$ оператор $\varphi(D)$ по формуле $(\varphi(D) f)(x)=\int_{\mathbb{R}} f(x+t) d \mu(t)$, где $\mu$ - любая мера из соответствующего символу $\varphi$ класса смежности $\mu_{\varphi}(K)$ в $M(\mathbb{R}) / J(K)$. Меру $\mu \in \mu_{\varphi}(K)$, для которой $\|\mu\|_{M(\mathbb{R})}=\left\|\mu_{\varphi}(K)\right\|_{M(\mathbb{R}) / J(K)}$, будем называть экстремальной. Следующая теорема содержится по существу в работах Бьёрлинга [3] и Горина [4], где она рассматривалась при разных дополнительных условиях на $K$.

Tеорема 1. Пусть $K-$ компакт в $\mathbb{R} u \varphi \in B(\mathbb{R}) / Z(K)$. Тогда $\|\varphi\|_{B(\mathbb{R}) / Z(K)}$ $=\left\|\mu_{\varphi}(K)\right\|_{M(\mathbb{R}) / J(K)}=\|\varphi(D)\|_{K}$, где $\|\varphi(D)\|_{K}-$ норма оператора $\varphi(D)$ в $B_{K}$. Оператор $\varphi(D)$ обладает в $B_{K}$ экстремалями, т. е. существуют такие $f \in B_{K}$, что $\|f\|=1 u(\varphi(D) f)(0)=\|\varphi(D)\|_{K}$. Мера $\mu \in \mu_{\varphi}(K)$ является экстремальной тогда и только тогда, когда $f \cdot \mu=|\mu|$ для каждой экстремали $f$, где $|\mu|$ - вариация мерь $\mu$.

Это утверждение сводит вычисление $\|\varphi\|_{B(\mathbb{R}) / Z(K)}$ к вычислению нормы оператора $\varphi(D)$ в $B_{K}$. Из него также следует, что если норма оператора $\varphi(D)$ в $B_{K}$ достигается уже на семействе $\left\{e^{i(\alpha x+\beta)}, \alpha \in K, \beta \in \mathbb{R}\right\}$, то в (1) оба неравенства превращаются в равенства. В противном случае имеет место неравенство

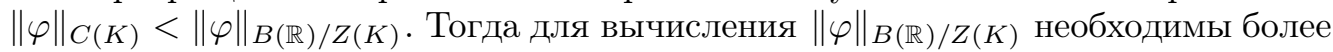
тонкие средства. Задача упрощается, если в $\mu_{\varphi}(K)$ имеются экстремальные меры. Тогда в некоторых случаях задачи описания экстремальных мер в $\mu_{\varphi}(K)$ и экстремалей оператора $\varphi(D)$ в $B_{K}$ оказываются эквивалентными. Рассмотрим такой случай.

Пусть $\varphi(t)=\sum_{k=0}^{n} c_{k} t^{k}, c_{k} \in \mathbb{C}$. Пусть $K=[-\sigma, \sigma], \sigma>0$. Известно, что если $\varphi(D)$ имеет в $B_{\sigma}=B_{[-\sigma, \sigma]}$ экстремаль $f$, для которой $|f(x)| \not \equiv 1, x \in \mathbb{R}$, то в 
$\mu_{\varphi}(K)$ существует единственная экстремальная мера $[3,5]$. Этот факт позволяет в следующих теоремах описать экстремали $\varphi(D)$ (с возможным последующим вычислением экстремальной меры и нормы $\left.\|\varphi\|_{B(\mathbb{R}) / Z(K)}\right)$.

Теорема 2. Пусть $f$ - экстремаль оператора $\varphi(D)$ в пространстве $B_{\sigma}$. Если $|f(x)| \not \equiv 1, x \in \mathbb{R}$, то существует такой полином $p$ без вещественных нулей степени $\operatorname{deg} p \leqslant \operatorname{deg} \varphi-1$, для которого функиия

$$
g(z)=\frac{\left(p f(z)+p^{*} f^{*}(z)\right)^{2}}{4 p p^{*}(z)}
$$

где $f^{*}(z)=\overline{f(\bar{z})}$, удовлетворяет уравнению экстремали

$$
u(z)\left(p p^{*}(z)\right)^{2}\left[g^{\prime}(z)\right]^{2}=4 \sigma^{2} v^{2}(z) g(z)[1-g(z)]
$$

здесъ $u$ - некоторый положительный, $а v$ - вещественный полиномы, для $\kappa о т о р ы х ~ \operatorname{deg} u \leqslant 2(\operatorname{deg} \varphi+\operatorname{deg} p-1), \operatorname{deg} v \leqslant \operatorname{deg} \varphi+3 \operatorname{deg} p-1 u \operatorname{deg} v=$ $2 \operatorname{deg} p+\frac{1}{2} \operatorname{deg} u$.

В следующей теореме используются те же обозначения, что и в теореме 2.

ТЕОРема 3. Если $f$ - экстремаль оператора $\varphi(D)$ в $B_{\sigma}$, mо

$$
f(x)=\frac{\sqrt{p p^{*}(x)}}{p(x)}\left(\sin (\sigma A(x))+i \frac{t(x)}{\sqrt{u(x)}} \cos (\sigma A(x))\right), \quad x \in \mathbb{R} ;
$$

здесь $t-$ некоторый вещественный полином $u \operatorname{deg} t \leqslant \operatorname{deg} \varphi+\operatorname{deg} p-1, \operatorname{deg} t \leqslant$ $\frac{1}{2} \operatorname{deg} u, \oint у н к u и и ~ \sqrt{p p^{*}} u \sqrt{u}-$ арифметические значения корней, $A-$ некоторая первообразная в $\mathbb{R}$ бункции $v /\left(p p^{*} \sqrt{u}\right)$, а старшие коэббициенты полиномов $и, v$ и рр* равны единице.

Теоремам о норме и экстремалях дифференциальных операторов в $B_{K}$, которые принято называть неравенствами Бернштейна, посвящена обширная литература. В [1] указано, что большинство известных точных неравенств соответствуют случаю равенства $\|\varphi(D)\|=\|\varphi\|_{C(K)}$ в $(1)$, т. е. норма таких $\varphi(D)$ достигается уже на экспоненциальных экстремалях. Наиболее общий из известных ранее результатов о полиномиальных неравенствах Бернштейна содержится в [6], где в $B_{\sigma}$ изучен случай произвольного эрмитова полинома $\varphi$, т. е. такого полинома $\varphi$, что $\varphi(-t)=\overline{\varphi(t)}, t \in \mathbb{R}$. Эквивалентная экстремальная задача в случае вещественных коэффициентов решена в [7] и [8]. Указанные выше теоремы 2 и 3 решают вопрос об уравнении и общем виде экстремалей для произвольного комплексного полиномиального оператора $\varphi(D)$ в $B_{\sigma}$, приведенный в сборнике нерешенных проблем [4].

\section{ЛитЕРАТУРА}

1. Горин E. A. Вестник Харьк. гос. унив., Прикл. матем. и мех., 45 (205), $77-$ 105 (1980). 2. König H. Arch. Math., 28 (4), 422-430 (1977). 3. Beurling A. Collected Works, Vol. 2, Birkhauser, Boston, 1989. 4. Gorin E. A. In: Lecture Notes in Math., Vol. 1043 (1984), pp. 646-651. 5. Крейн М. Г. Уч. зап. Куйбышевского пед. ин-та, 7, 123-147 (1943). 6. Boas R. P., Shaeffer A. C. Amer. J. Math., 79, 857-884 (1957). 7. Ахиезер Н. И. Теория функций, функц. анализ и их прил., 1, 111-135 (1965). 8. Норвидас C. Литовский матем. сб., 38 (2), 248-259 (1998).

Институт математики и информатики, Вильнюс, Литва e-mail: norvidas@ieva.mif.vu.lt 